\title{
Influence of the Angular Distribution Function of Incident Particles on the Microstructure and Anomalous Scaling Behavior of Thin Films
}

\author{
A. Yanguas-Gil,,${ }^{1, *}$ J. Cotrino, ${ }^{1,2}$ A. Barranco, ${ }^{1,3}$ and A. R. González-Elipe ${ }^{1}$ \\ ${ }^{1}$ Instituto de Ciencia de Materiales de Sevilla (CSIC - University of Sevilla), Av Américo Vespucio 49, 41092 Sevilla, Spain \\ ${ }^{2}$ Departamento de Física Atómica, Molecular y Nuclear, Av Américo Vespucio 49, 41092 Sevilla, Spain \\ ${ }^{3}$ Departamento de Química Inorgánica (University of Sevilla), Av Américo Vespucio 49, 41092 Sevilla, Spain
}

(Received 16 December 2005; published 14 June 2006)

\begin{abstract}
The microstructure and the scaling properties of films grown by plasma enhanced chemical vapor deposition are reproduced with a discrete model that takes into account the angular distribution function of the particles and the lateral growth of the films. Both the experimental and simulated surfaces exhibit a granular microstructure and an anomalous scaling behavior characterized by values of the growth exponent $\beta$ that vary with the scale of measurement. Depending on the angular distribution function used in the model, values of $\beta$ ranging from 0.86 to 0.2 are obtained.
\end{abstract}

DOI: 10.1103/PhysRevLett.96.236101

Rough surfaces and interfaces are ubiquitous in nature, and from the technological point of view the control of their roughness is becoming critical for applications in fields such as microelectronics, image formation, surface coating, or thin film growth [1]. In this context, during the last years there has been an increasing interest in the description of the self-affine kinetic roughening of surfaces [2-6]. This regime is characterized by an increase of the roughness with time and/or the scale of observation in a self-affine fashion $[7,8]$. The conventional dynamic scaling theory, the theoretical background used for the description of these phenomena, is based on the so-called FamilyVicsec relation, which models the evolution of the surface roughness $\sigma$-defined as the rms value of the heights of the different points of the interface- with the deposition time and the scale of measurement by means of the following expression [8]:

$$
\sigma(L, t) \sim L^{\alpha} f\left(\frac{t}{L^{\alpha / \beta}}\right) \begin{cases}f(u) \sim u^{\beta} & u \ll 1 \\ f(u) \equiv \text { const } & u \gg 1\end{cases}
$$

where $\alpha$ is the so-called roughness exponent and $\beta$ the growth exponent. According to the dynamic scaling theory, there exist only a finite number of universality classes characterized by certain values of the $(\alpha, \beta)$ exponents, so that all the experimental systems would fall into one of these classes. The dynamic scaling theory can then be understood as an extension of the theoretical framework used for the description of the scaling properties of equilibrium phase transitions to nonequilibrium systems [7].

In the last years a number of experimental results have appeared that cannot be interpreted in terms of the conventional dynamic scaling theory. In some works, values of the $\alpha$ and/or $\beta$ exponents which do not belong to any of the universality classes have been reported. An example of this behavior is the finding of very high values of the growth exponent $\beta$ (i.e., $\beta>0.5$ ) [2,9]. To account for these values, alternative explanations of that of the universality classes have been proposed. One of the most widely used
PACS numbers: 68.55. $-\mathrm{a}, 64.60 . \mathrm{Ht}, 68.47 .-\mathrm{b}, 81.15 . \mathrm{Gh}$

models is the nondeterministic model of Das SarmaTamborenea [10], that relates the variations of the growth exponent $\beta$ with the control of film growth by surface diffusion. For 2D surfaces, this model accounts for values of this exponent ranging from 0.17 to 0.5 , this latter value being only obtained if diffusion is neglected. In the same context, Elsholtz et al. [9] presented a model where they considered a diffusion energy with two contributions, one determined by the bonding environment of the incoming particles once they arrive to the surface, and a second one of stochastic character. By changing the relative weight of the stochastic parameter, these authors were able to obtain values of the $\beta$ exponent as high as 1.0.

In other systems, the scaling of surface roughness is characterized by different local and global behaviors (i.e., at short and long length scales $L$ ) of the surface roughness [11-14], a result that precludes the use of the FamilyVicsec relation [6]. In order to explain the anomalous scaling observed, some authors have defined different scaling relations for the local and global surface fluctuations [6], while other authors have developed modified scaling relations where the local and global behaviors are gathered in a single expression [13]. However, the physical interpretation of the anomalous scaling and its relation with the kinetics of the growth process is not straightforward and there is a strong need of more elaborated models for a better understanding of the roughening process.

In this work, we present new experimental results that reveal an anomalous scaling of the surface roughness of $\mathrm{SiO}_{x} \mathrm{C}_{y} \mathrm{H}_{z}$ and $\mathrm{SiO}_{2}$ thin films prepared by plasma enhanced chemical vapor deposition (PECVD) [15]. We show that the anomalous scaling behavior and the surface microstructure can be reproduced by a kinetic Monte Carlo simulation in which the effect of the angular distribution function of the incoming particles and the presence of lateral growth are specifically taken into account as the main factors responsible for the control of the growth process. Despite the fact that the model primarily intends 
to describe thin film growth in reactive environments characteristic of PECVD, we believe that it can be also applied to any other system in which diffusion does not play a significant role.

$\mathrm{SiO}_{x} \mathrm{C}_{y} \mathrm{H}_{z}$ and $\mathrm{SiO}_{2}$ thin films have been prepared by PECVD in a low pressure plasma reactor by using tetramethylsilane as the precursor and mixtures of $\mathrm{Ar} / \mathrm{O}_{2}$ as plasma gas. It is interesting to mention that although different compositions (i.e., Si:C:O relations) can be obtained depending on the actual $\mathrm{Ar} / \mathrm{O}_{2}$ ratio in the plasma, the scaling behavior of the films was always similar.

Figure 1 shows the most significant experimental results regarding the film microstructure and the scaling behavior of the surfaces of the $\mathrm{SiO}_{x} \mathrm{C}_{y} \mathrm{H}_{z}$ and $\mathrm{SiO}_{2}$ films. The films depict a well-defined granular microstructure and are characterized by high values of the growth exponent (i.e., $\beta \approx$ 0.57 for $\mathrm{SiO}_{2}$ and $\beta \approx 0.58$ for $\mathrm{SiO}_{x} \mathrm{C}_{y} \mathrm{H}_{z}$ at large scales of measurement). Also, values of $\alpha \sim 1.3$ have been determined by means of the power spectral density for global scaling [2,11,16], while values of $\alpha \sim 0.7$ have been found by using the height-height correlation function for local scaling [17]. However, the most interesting result in relation with the anomalous behavior of these surfaces is the

a)

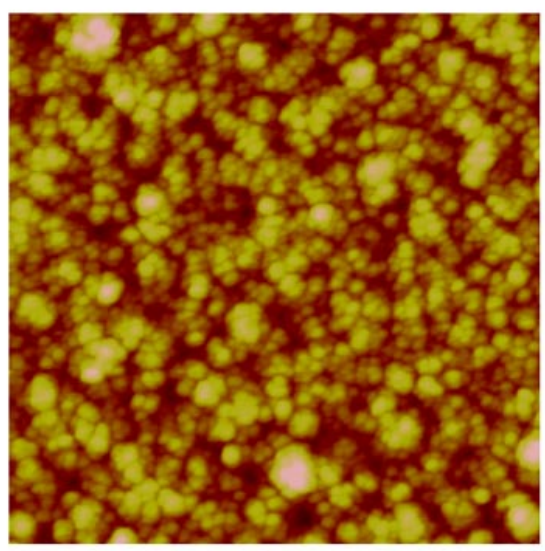

b)

$\sigma=2.84 \mathbf{n m}$

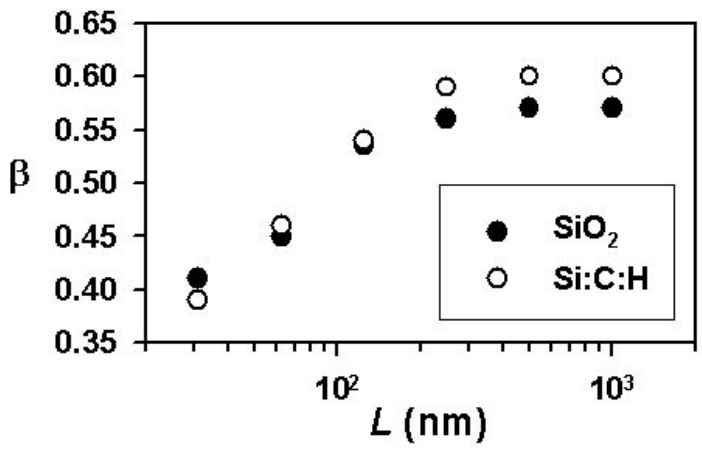

FIG. 1 (color online). $\quad \mathrm{SiO}_{x} \mathrm{C}_{y} \mathrm{H}_{z}$ and $\mathrm{SiO}_{2}$ thin films grown by PECVD: (a) Typical atomic force microscope image of a $\mathrm{SiO}_{2}$ thin film ( $1 \times 1$ microns); (b) Variation of the growth exponent $\beta$ with the scale of measurement of $\mathrm{SiO}_{2}$ and $\mathrm{SiO}_{x} \mathrm{C}_{y} \mathrm{H}_{z}$ thin films. dependence found between the growth exponent $\beta$ and $L$. This relation, also reported in Fig. 1, clearly evidences an important decrease of the $\beta$ exponent for low values of $L$. This result would be in agreement with the existence of a characteristic length or size $\left(L_{c}\right)$ of the system. For $L>L_{c}$ a constant global exponent is found, while for $L<L_{c}$ a variable local value of the $\beta$ exponent is obtained. From the results in Fig. 1, in our system this would occur for $L_{c} \sim 100 \mathrm{~nm}$. Therefore, our system presents an anomalous scaling behavior that cannot be explained in terms of the Family-Vicsec relation.

To understand the scaling properties and the microstructure developed by systems depicting an anomalous scaling behavior [11-14] and, in particular, our own PECVD films, the film growth has been modeled by means of a kinetic Monte Carlo simulation. In this regard it is interesting to note some characteristic features of the PECVD procedure such as the existence of reactive species in the gas phase and dangling bonds at the surface. These dangling bonds may hinder the surface diffusion and thus contribute to the appearance of vacancies, the development of microporosity, and the decrease of the density of the films. In the model, we first admit that the incoming particles may reach the surface with an angular distribution function. This is constructed by considering, as in the work of Karabacak et al. [18], a Maxwellian distribution function for the energy of the particles. In addition, we also include an average directed velocity $v_{0}$ towards the surface. When this directed velocity is small, the angular distribution function spreads the incoming direction of the particles, a situation that is consistent with the physical characteristics of a plasma [19]. Meanwhile, when $v_{0}$ is dominant, particles reach the surface with the same angle, a situation close to that existing in "line of sight" techniques like physical vapor deposition or molecular beam epitaxy, where the incoming particles likely follow straight trajectories.

With this model it is possible to obtain an analytical expression for the accumulated distribution function $F(\Theta)=P[\theta<\Theta]$, that gives the probability for a particle to reach the surface with an angle smaller than $\Theta$ with respect to the normal direction of the surface:

$$
P[\theta<\Theta]=1-\cos \Theta \frac{\exp \left(z^{2} \cos ^{2} \Theta\right)[1+\operatorname{erf}(z \cos \Theta)]}{\exp \left(z^{2}\right)[1+\operatorname{erf}(z)]},
$$

with $z$ being a normalized velocity given by

$$
z^{2}=\frac{M v_{0}^{2}}{2 k_{B} T}
$$

Therefore, depending on the value of the normalized velocity $z$, we can simulate situations ranging from nearly vertical incidence of the particles to a pure Maxwellian distribution of the incoming particles with a large span of angles of incidence.

A second important assumption of the model is that we have abandoned the solid-on-solid approximation and al- 


2. $z=0 \quad z=1.5 \quad z=2$

FIG. 2. Representation of the topography images for simulated thin films of equivalent thickness, showing the influence of the angular distribution function of the incoming particles on the surface microstructure.

lowed the system to form voids and overhangs during the film growth. It is assumed that, as in the ballistic deposition model $[8,20]$, particles stick initially onto the first position where they touch the surface. This assumption is consistent with the fact that in PECVD the incoming particles usually have an enhanced reactivity and the obtained materials present strong covalent and ionic bonds. From this state, surface diffusion is only allowed to single bonded particles. This restriction is in agreement with the low temperatures characteristic of the PECVD method, whereby the strong surface binding energies (i.e., $\mathrm{Si}-\mathrm{O}$ and $\mathrm{Si}-\mathrm{C}$ bonds in our films) will greatly decrease the diffusion frequency of multiple bonded atoms. Note that although the model has been developed to reproduce the conditions encountered by PECVD, it basically consists of a ballistic deposition model with particles arriving with an angular distribution that, once on the surface, present a restricted or null diffusion. In this sense, the model represents a general situation in which lateral growth and nonlocal interactions due to shadowing effects are present.

Simulations have been performed for $N \times N$ particles, with $N$ ranging from 128 to 2056 . For each condition $20-$ 100 simulations have been run to improve the statistics. Figure 2 shows the influence of the normalized directed velocity $z$ on the film microstructure in the absence of diffusion. The images correspond to the theoretical simulation of the growth of surfaces composed by $256 \times 256$ active sites and similar optical thickness. It is apparent that the distribution function of the incident particles greatly affects the microstructure of the simulated films, and that the granular structure found experimentally is described best by a situation characterized by a low diffusion and relatively small values of the directed velocities of the incoming particles $(z \ll 1)$. Under these conditions, a net increase in grain size with deposition time is a direct consequence of the formation of voids and overhangs. For the case $z=0$, Fig. 3 shows that the coalescence of the growing grains occurs with time, thus avoiding the development of columns in the films characteristic of situations in which, as in solid-on-solid approximations, the role of lateral growth is minimized [21].

The variation of film roughness with deposition time is presented in Fig. 4 for $N=256$ and different values of $z$. Apart from the expected transient region at low thickness $(h<10)$ where the growth exponent is close to 0.5 [8], the films are characterized by different values of the $\beta$ expo-

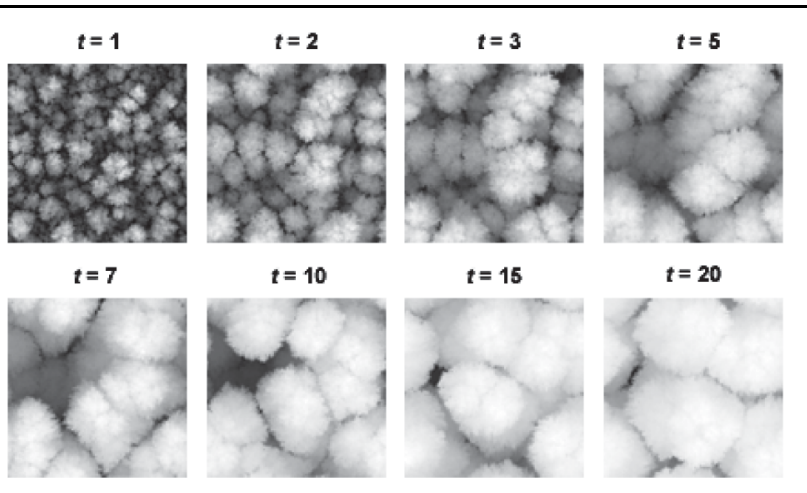

FIG. 3. Evolution of the simulated surface topography with deposition time $(z=0)$.

nent before reaching saturation. The plot also shows that the exponent $\beta$ is strongly influenced by the distribution function of the incoming particles, reaching a maximum value for a pure Maxwellian angular distribution function $(z=0)$.

When analyzing the time dependence of the roughness for different length scales, a strong dependence of the $\beta$ value with the scale of measurement is found, especially at low length scales. Thus Fig. 5(a) shows that for regions below the correlation length (i.e., $L_{c}(N) \sim 500$ ), $\beta$ changes with $N$. It is also interesting in this figure that the magnitude of the change of $\beta$ critically depends on the angular distribution function of the particles. For $N>500$ a saturation value is obtained for $\beta$, in perfect agreement with the experimental results presented in Fig. 1. At this point it is important to note that, as shown in Fig. 4, the scaling relation $\sigma \sim t^{\beta}$ still holds for low length scales (i.e., $N=256$ for the plots in Fig. 4). This means that the

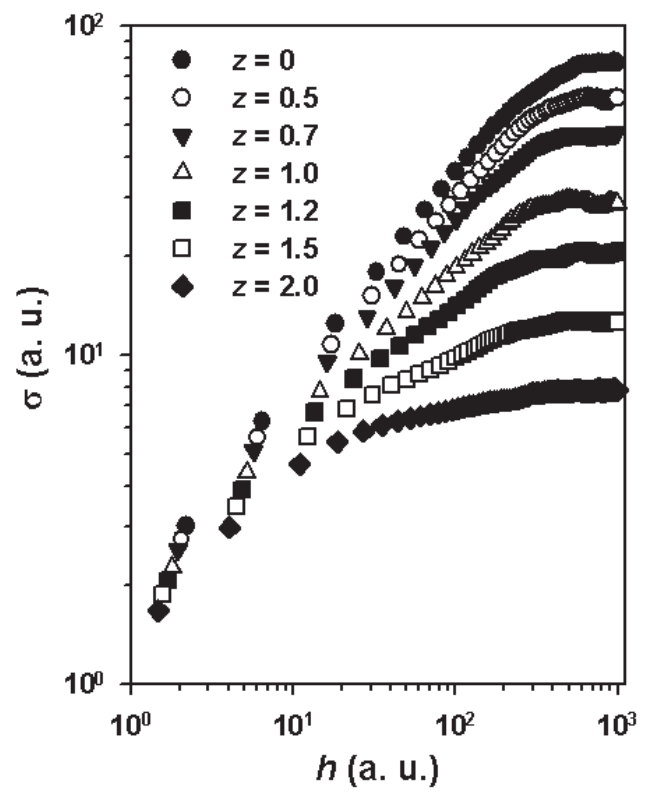

FIG. 4. Evolution of the calculated surface roughness as a function of the film thickness for different values of the normalized directed velocity $z(N=256)$. 

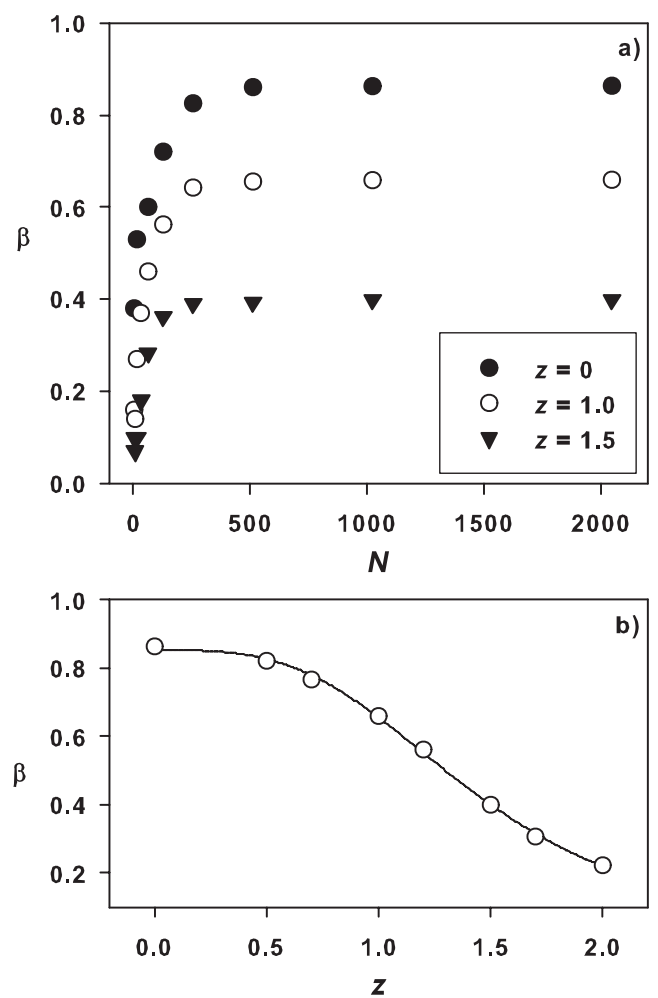

FIG. 5. Evolution of the growth exponent $\beta$ with the normalized directed velocity $z$ and the scale length of the system: (a) Variation of $\beta$ with the scale length for selected $z$ values; (b) Influence of the distribution function of the incoming particles on the saturation values of the growth exponent.

decrease of the $\beta$ exponent presented in Fig. 5(a) cannot be interpreted as a transient region between the two asymptotic regimes of the Family-Vicsec relation, but as a scaling property of the roughness of the simulated film. Such a behavior can be considered a generalization of the scaling relation proposed by Huo and Schwarzacher [13].

The saturation values of $\beta$ obtained for high length scales are presented in Fig. 5(b) for different values of $z$. This plot shows that by changing the angular distribution function of the particles it is possible to obtain a wide range of values of the growth exponent, from $\beta=0.82$ to $\beta=$ 0.2 for an almost perpendicular incidence of the incoming particles. Therefore, the proposed model furnishes a wide range of growth exponents and final microstructures that agree not only with the experimental measurements presented here for PECVD films, but also with those of other authors for thin films grown by other methods $[2,9]$.

Although further work is still necessary to fully understand the effect of surface diffusion in the transition from a granular to a columnar microstructure and its influence on the scaling properties of the growing surface, the proposed model proves that both the angular distribution of the incoming particles and lateral growth can play an important role in determining the microstructure and the scaling properties of surfaces during thin film growth. The presence of an angular distribution function of the incoming particle introduces nonlocal effects in the surface dynamics that would be the main reason for the anomalous behavior of the scaling properties of the surface as evidenced by the high values of the $\beta$ exponent determined. Lateral growth, on the other hand, would be responsible for the appearance of a granular microstructure that would prevent the development of the columnar microstructure characteristic of the solid-on-solid approximations.

*Electronic address: angel.yanguas@icmse.csic.es. Present address: Fakultät für Physik und Astronomie, Ruhr Universität, 44780 Bochum, Germany.

[1] T.S. Chow, Mesoscopic Physics of Complex Materials, Graduate Texts in Contemporary Physics (Springer Verlag, Berlin, 2000).

[2] F. Ojeda, R. Cuerno, R. Salvarezza, and L. Vazquez, Phys. Rev. Lett. 84, 3125 (2000).

[3] K. R. Bray and N. G. Parsons, Phys. Rev. B 65, 035311 (2002).

[4] T. Karabacak, Y.-P. Zhao, G.-C. Wang, and T. M. Lu, Phys. Rev. B 66, 075329 (2002).

[5] A. C. Durr, F. Schreiber, K. A. Ritley, V. Kruppa, J. Krug, H. Dosch, and B. Struth, Phys. Rev. Lett. 90, 016104 (2003).

[6] J. M. Lopez, M. Castro, and R. Gallego, Phys. Rev. Lett. 94, 166103 (2005).

[7] G. Odor, Rev. Mod. Phys. 76, 663 (2004).

[8] A.L. Barabasi and H.E. Stanley, Fractal Concepts in Surface Growth (Cambridge University Press, Cambridge, 1995).

[9] F. Elshotz, E. Schöll, and A. Rosenfeld, Appl. Phys. Lett. 84, 4167 (2004).

[10] S. Das Sarma and P. Tamborenea, Phys. Rev. Lett. 66, 325 (1991).

[11] A. E. Lita and J.E. Sanchez, Jr., Phys. Rev. B 61, 7692 (2000).

[12] N. M. Hasan, J. J. Mallett, S. G. dos Santos Filho, A. A. Pasa, and W. Schwarzacher, Phys. Rev. B 67, 081401 (2003).

[13] S. Huo and W. Schwarzacher, Phys. Rev. Lett. 86, 256 (2001).

[14] M. Saitou, Phys. Rev. B 66, 073416 (2002).

[15] A. Barranco, J. Cotrino, F. Yubero, and A. R. GonzalezElipe, Chem. Mater. 15, 3041 (2003).

[16] S. Vauth, C. Streng, S. G. Mayr, and K. Samwer, Phys. Rev. B 68, 205425 (2003).

[17] J. H. Jeffries, J.-K. Zuo, and M. M. Craig, Phys. Rev. Lett. 76, 4931 (1996).

[18] T. Karabacak, Y.-P. Zhao, G.-C. Wang, and T. M. Lu, Phys. Rev. B 64, 085323 (2001).

[19] D. J. Economou, T.S. Bartel, R.S. Wise, and D.P. Lymberopoulos, IEEE Trans. Plasma Sci. 23, 581 (1995).

[20] J. Yu and J. G. Amar, Phys. Rev. E 65, 060601(R) (2002).

[21] J. T. Drotar, Y.-P. Zhao, T.-M. Lu, and G.-C. Wang, Phys. Rev. B 62, 2118 (2000). 